\title{
PREVALENCIA DE CARIES NO TRATADA EN NIÑOS ECUATORIANOS EN RIESGO SOCIAL: ESTUDIO COMPARATIVO
}

\section{Prevalence of untreated dental caries in ecuadorian children in social risk: comparative study}

\author{
Oliván-Gonzalvo Gonzalo¹, Oliván-Gracia Santiago², De la Parte-Serna Alejandro Carlos ${ }^{3}$ \\ ${ }^{1}$ Especialista en Pediatría. Instituto Aragonés de Servicios Sociales. Zaragoza, España. \\ ${ }^{2}$ Grado en Medicina. Universidad de Zaragoza. Zaragoza, España. \\ ${ }^{3}$ Profesor de Odontología. Universidad de Zaragoza. Zaragoza, España.
}

DOI: https://doi.org/10.31984/oactiva.v6i3.454

\section{Resumen}

\begin{abstract}
Objetivo: Determinar la prevalencia de caries no tratada en niños ecuatorianos acogidos en el sistema de protección de Aragón (España). Observar si existe asociación con el índice de masa corporal (IMC) y la situación de desprotección. Observar diferencias con niños de otros países latinoamericanos en la misma situación. Materiales y métodos: Estudio transversal sobre 59 niños ecuatorianos y 175 de otros países latinoamericanos de 6-17 años de edad. Se definieron las situaciones de desprotección y se realizó exploración antropométrica y bucodental estandarizada. Para el estudio comparativo se utilizaron la prueba $\mathrm{Z}$ y el T-test con un nivel de significación de $\mathrm{p}<0,05$. Resultados: La prevalencia de caries no tratada en los niños ecuatorianos fue de $25,4 \%$, significativamente superior $(\mathrm{p}=0,366)$ a la de los niños de otros países latinoamericanos. Existió asociación positiva $(p=0,003)$ con la situación de imposibilidad parental temporal o definitiva para ejercer los deberes de protección. No se observó asociación con el estado nutricional de acuerdo con el IMC. Conclusiones: Consideramos que, además de los hábitos dietéticos cariogénicos nativos, la situación de desprotección y deprivación sociofamiliar es un factor esencial en la alta prevalencia de caries no tratada observada en los niños ecuatorianos acogidos en el sistema de protección.
\end{abstract}

Palabras clave: Prevalencia, caries, Ecuador, Latinoamérica, índice de masa corporal, maltrato infantil.

\begin{abstract}
Objective: To determine the prevalence of untreated dental caries in Ecuadorian children in the Aragón protection system (Spain). Observe whether there is an association with the body mass index (BMI) and the situation of lack of protection. Observe differences with children from other Latin American countries in the same situation. Materials and methods: Cross-sectional study on 59 Ecuadorian children and 175 from other Latin American countries 6-17 years old. Lack of protection situations were defined, and standardized anthropometric and oral examination was performed. The $Z$ test and the T-test with a significance level of $p<0.05$ were used for the comparative study. Results: The prevalence of untreated dental caries in Ecuadorian children was $25.4 \%$, significantly higher $(p=0.366)$ than that of children in other Latin American countries. There was a positive association $(p=0.003)$ with the situation of temporary or permanent parental impossibility to exercise the duties of protection. No association was observed with nutritional status according to BMI. Conclusions: We consider that, in addition to the native cariogenic dietary habits, the situation of socio-family lack of protection and deprivation is an essential factor in the high prevalence of untreated dental caries observed in Ecuadorian children in the protection system.
\end{abstract}

Key words: Prevalence, caries, Ecuador, Latin America, body mass index, child abuse.

\section{Introducción}

En los países de la Región Europea de la Organización Mundial de la Salud (OMS) la salud bucodental se ha reconocido como más pobre en la población migrante que en la población de acogida y la atención dental se considera uno de los problemas clave entre la población infanto-juvenil migrante. ${ }^{1}$
España es el cuarto país de la Unión Europea que alberga a la mayor cantidad de inmigrantes en los últimos 25 años, por detrás de Alemania, Reino Unido y Francia ${ }^{2}$. Los inmigrantes residentes en España (definidos por su origen y no por acceso a la nacionalidad española) representan actualmente el $14 \%$ del total de la población y el $6 \%$ de la población entre $0-15$ años. Los originarios de Latinoamérica son el segundo colectivo más numeroso, por detrás de los originarios de otros países de la Unión Europea. Entre 
los inmigrantes latinoamericanos el colectivo más numeroso es el originario de Ecuador, seguido de los de Colombia, Perú, Brasil y Venezuela. En la Comunidad Autónoma de Aragón el colectivo más numeroso también es el originario de Ecuador, seguido de los de Colombia, Brasil, Perú y Venezuela. ${ }^{3}$

Según la Federación Dental Internacional (FDI) la caries es la enfermedad crónica más extendida en el mundo. Se debe principalmente a la exposición al azúcar, además de otros factores de riesgo, y a la falta de prevención efectiva y el acceso limitado a los cuidados bucodentales ${ }^{4}$. Los expertos también vinculan el exceso de consumo de azúcar con el desarrollo de sobrepeso y obesidad en la edad pediátrica. $^{5}$

Entre otros factores de riesgo de caries, se ha observado asociación con la vulnerabilidad y deprivación social y que son numerosos los factores ambientales, familiares e individuales que contribuyen a la alta prevalencia de dientes cariados no tratados en los niños y adolescentes maltratados o en situación de riesgo social. ${ }^{6,7}$ En España del total de niños atendidos en el sistema de protección mediante acogimiento residencial durante el periodo 2015-2017 por encontrase en situación de riesgo social, el $33 \%$ son inmigrantes. Esta cifra es 5 veces superior a la de la población infantil inmigrante residente en España ${ }^{8}$.

Los objetivos de este estudio son:

- Determinar la prevalencia de caries no tratada en una cohorte de niños ecuatorianos acogidos residencialmente en el sistema de protección de niños de Aragón (España) por encontrarse en situación de riesgo social.

- Realizar un estudio comparativo con una cohorte de niños originarios del conjunto de otros países latinoamericanos con la misma problemática.

- Observar si existen asociaciones con el índice de masa corporal (IMC) y las situaciones de desprotección.

\section{Materiales y métodos}

Estudio observacional y descriptivo de diseño transversal y retrospectivo sobre una cohorte de 59 niños ecuatorianos y 175 niños originarios de otros países latinoamericanos (Colombia, 43; República Dominicana, 34; Brasil, 27; Venezuela, 14; Nicaragua, 13; Perú, 10; Cuba, 9; Honduras, 8; Argentina, 8; Paraguay, 3; Guatemala, 2; Bolivia, 1; Chile, 1; El Salvador, 1; Panamá, 1), con un rango de edad entre 6-17 años, que fueron acogidos residencialmente en el sistema de protección de niños de la Comunidad Autónoma de Aragón (España) durante el periodo 2000-2019.

Las situaciones de desprotección se definieron utilizando el protocolo estandarizado Instrumento para la Valora- ción de la Gravedad de las Situaciones de Desprotección Infantil en Aragón-20149 . Entre las variables demográficas estudiadas, se analizaron subgrupos de edad y se definieron los diferentes subgrupos generacionales: hijos de inmigrantes nacidos en España (segunda generación) e hijos de inmigrantes nacidos en origen llegados a España con edades entre 0-5 años (generación 1.75), entre 6-12 años (generación 1.5), y entre 13-17 años (generación 1.25). ${ }^{10}$

La exploración antropométrica y bucodental se realizó de forma protocolizada en el momento del acogimiento. En la exploración antropométrica, el peso se registró como la media de dos determinaciones usando una báscula electrónica de columna seca 799, con una precisión de 100 g, y la talla se registró como la media de dos mediciones con un tallímetro incorporado a la báscula con el menor en posición erecta hasta el milímetro más cercano. Todas las mediciones fueron realizadas por el mismo explorador. Para definir el sobrepeso y la obesidad se calculó el IMC (peso/talla al cuadrado) y para interpretar su valor se utilizaron los puntos de corte específicos para edad y sexo elaborados por Cole et al. y propuestos por la International Obesity Task Force. ${ }^{11}$ En la exploración bucodental se utilizó el protocolo y el formulario estandarizado de la $\mathrm{OMS}^{12}$ y la nomenclatura dental conforme al código adoptado por la $\mathrm{FDI}^{4}$.

Análisis estadístico. Se utilizó una hoja Excel® para calcular la media aritmética y desviación estándar (DE) de la edad y el IMC de los niños y los porcentajes de las variables estudiadas. Los análisis estadísticos se realizaron con el programa Social Science Statistics ${ }^{\circledR}$. Para la comparación de medias independientes se utilizó la prueba T-test, de dos colas, con un nivel de significación de $\mathrm{p}<0,05$. Para la comparación de proporciones poblacionales se utilizó la prueba $\mathrm{Z}$, de dos colas, con un nivel de significación de $\mathrm{p}<$ 0,05 .

\section{Resultados}

En la Tabla 1 se detallan y se comparan las variables demográficas de las muestras de niños ecuatorianos y del conjunto de otros países latinoamericanos acogidos en el sistema de protección de Aragón. No se observaron diferencias significativas en cuanto a edad y sexo. Respecto a los subgrupos de edad, la proporción de niños de 6-12 años fue significativamente mayor en los ecuatorianos ( $\mathrm{p}=$ $0,024)$, mientras que los de 13-17 años fue significativamente mayor en el conjunto de otros países latinoamerica$\operatorname{nos}(\mathrm{p}=0,024)$. Respecto a los subgrupos generacionales, sólo el 6,8\% y el $8,6 \%$, respectivamente, pertenecían a la segunda generación. Se observó diferencia estadísticamente significativa a favor de los ecuatorianos en la generación 1.75 ( $\mathrm{p}<0,001)$, y a favor del conjunto de otros países latinoamericanos en la generación $1.25(\mathrm{p}=0,324)$. 
Tabla 1. Variables demográficas de los niños ecuatorianos y del conjunto de otros países latinoamericanos en el sistema de protección de Aragón (España)

\begin{tabular}{|c|c|c|}
\hline & $\frac{\text { Ecuador }}{(n=59)}$ & $\frac{\begin{array}{c}\text { Otros países } \\
\text { Latinoamericanos }\end{array}}{(n=175)}$ \\
\hline \multicolumn{3}{|l|}{ Variables demográficas } \\
\hline Varón [n (\%)] & $25(42,4)$ & $78(44,6)$ \\
\hline Mujer [n (\%)] & $34(57,6)$ & $97(55,4)$ \\
\hline Edad [años; media (DE)] & $12,7(4,1)$ & $13,9(3,6)$ \\
\hline Edad 6-12 años [n (\%)] & $27(45,8)^{*}$ & $52(29,7)$ \\
\hline Edad 13-17 años [n (\%)] & $32(54,2)$ & $123(70,3)^{*}$ \\
\hline Generación 2 [n (\%)] & $4(6,8)$ & $15(8,6)$ \\
\hline Generación 1.75 [n (\%)] & $24(40,7)^{*}$ & $28(16)$ \\
\hline Generación 1.5 [n (\%)] & $24(40,7)$ & $88(50,3)$ \\
\hline Generación 1.25 [n (\%)] & $7(11,9)$ & $44(25,1)^{*}$ \\
\hline
\end{tabular}

En la Tabla 2 se detalla y se compara la prevalencia de caries no tratada, de sobrepeso y obesidad, y de la situación de desprotección en las muestras de niños ecuatorianos y del conjunto de otros países latinoamericanos acogidos en el sistema de protección de Aragón. La prevalencia de caries no tratada fue significativamente superior en los niños ecuatorianos $(p=0,366)$, siendo casi el doble que la observada en los niños del conjunto de otros países latinoamericanos. No se observaron diferencias respecto al estado nutricional de acuerdo con el IMC. En los niños ecuatorianos las situaciones de desprotección significativamente más prevalentes fueron el maltrato físico $(\mathrm{p}<0,001)$ y la existencia de dos o más situaciones $(\mathrm{p}<0,001)$. En los niños del conjunto de otros países latinoamericanos la situación de desprotección significativamente más prevalente fue la incapacidad parental de control de la conducta del menor $(\mathrm{p}<0,001)$.

Tabla 2. Prevalencia de caries no tratada, sobrepeso/obesidad y situaciones de desprotección en los niños ecuatorianos y del conjunto de otros países latinoamericanos en el sistema de protección de Aragón (España)

\begin{tabular}{lcc}
\hline & $\frac{\text { Ecuador }}{(\mathbf{n}=59)}$ & $\begin{array}{c}\text { Otros países } \\
\text { Latinoamericanos }\end{array}$ \\
Caries no tratada [n (\%)] & $15(25,4)$ & $24(13,7)$ \\
IMC $\left[\mathrm{kg} / \mathrm{m}^{2} ;\right.$ media $\left.(\mathrm{DE})\right]$ & $21,6(4,1)$ \\
Normal [n (\%)] & $21,1(4,9)$ & $148(84,6)$ \\
Sobrepeso [n (\%)] & $51(86,4)$ & $17(9,7)$ \\
Obesidad [n (\%)] & $4(6,8)$ & $10(5,7)$ \\
Sobrepeso + Obesidad [n (\%)] & $4(6,8)$ & $27(15,4)$ \\
Situación de desprotección [n (\%)] & $8(13,6)$ & \\
Incapacidad parental de control de la & & $68(38,9)$ \\
$\quad$ conducta del menor & $8(13,6)$ & $33(18,9)$ \\
Maltrato físico & $36(61) *$ & $24(13,7)$ \\
Negligencia física & $10(16,9)$ & \\
Imposibilidad parental temporal o definitiva & & $28(16)$ \\
para ejercer los deberes de protección & $7(11,9)$ & $19(10,9)$ \\
Maltrato psicológico & $11(18,6)$ & $17(9,7)$ \\
Abandono & $3(5,1)$ & $6(3,4)$ \\
Abuso sexual & $3(5,1)$ & $17(9,7)$ \\
Dos o más situaciones de desprotección & $17(28,8) *$ & \\
& &
\end{tabular}

$* p<0,05$
En la Tabla 3 se compara la presencia o ausencia de caries no tratada con las variables demográficas, el IMC y las situaciones de desprotección en la muestra de niños ecuatorianos en el sistema de protección de Aragón.

La presencia de caries no tratada fue significativamente superior en el sexo femenino $(p=0,042)$ y en los niños cuya situación de desprotección fue la imposibilidad parental temporal o definitiva para ejercer los deberes de protección $(\mathrm{p}=0,003)$

Tabla 3. Comparación de la presencia/ausencia de caries no tratada con el IMC y la situación de desprotección en los niños ecuatorianos en el sistema de protección de Aragón (España)

\begin{tabular}{|c|c|c|}
\hline & \multicolumn{2}{|c|}{ Ecuador } \\
\hline & $\frac{\text { Caries no tratada }(+)}{(n=15)}$ & $\frac{\text { Caries no tratada }(-)}{(n=44)}$ \\
\hline \multicolumn{3}{|l|}{ Variables demográficas [n (\%)] } \\
\hline Varones & $3(20)$ & $22(50)$ \\
\hline Mujeres & $12(80)^{*}$ & $22(50)$ \\
\hline Edad 6-12 años & $9(60)$ & $18(40,9)$ \\
\hline Edad 13-17 años & $6(40)$ & $26(59,1)$ \\
\hline Generación 2 & $2(13,3)$ & $2(4,5)$ \\
\hline Generación 1.75 & $8(53,3)$ & $16(36,4)$ \\
\hline Generación 1.5 & $4(26,7)$ & $20(45,5)$ \\
\hline Generación 1.25 & $1(6,7)$ & $6(13,6)$ \\
\hline \multicolumn{3}{|l|}{$\operatorname{IMC}[\mathrm{n}(\%)]$} \\
\hline Normal & $14(93,3)$ & $37(84,1)$ \\
\hline Sobrepeso & $0(0)$ & $4(9,1)$ \\
\hline Obesidad & $1(6,7)$ & $3(6,8)$ \\
\hline Sobrepeso + Obesidad & $1(6,7)$ & $7(15,9)$ \\
\hline \multicolumn{3}{|l|}{ Situación de desprotección [n (\%)] } \\
\hline \multicolumn{3}{|l|}{ Incapacidad parental de control de la | } \\
\hline conducta del menor & $1(6,7)$ & $7(15,9)$ \\
\hline Maltrato fisico & $6(40)$ & $30(68,2)^{*}$ \\
\hline Negligencia física & $2(13,3)$ & $8(18,2)$ \\
\hline \multicolumn{3}{|l|}{ Imposibilidad parental temporal o definitiva } \\
\hline para ejercer los deberes de protección & $5(33,3)^{*}$ & $2(4,5)$ \\
\hline Maltrato psicológico & $2(13,3)$ & $9(20,5)$ \\
\hline Abandono & $1(6,7)$ & $2(4,5)$ \\
\hline Abuso sexual & $0(0)$ & $3(6,8)$ \\
\hline Dos o más situaciones de desprotección & $2(13,3)$ & $15(34,1)$ \\
\hline
\end{tabular}

${ }^{*} p<0,05$

En la Tabla 4 se compara la presencia o ausencia de caries no tratada con las variables demográficas, el IMC y las situaciones de desprotección en la muestra de niños del conjunto de países latinoamericanos en el sistema de protección de Aragón.

La presencia de caries no tratada fue significativamente superior en el grupo de edad 6-12 años ( $p<0,001)$, en los pertenecientes a la segunda generación $(\mathrm{p}=0,021)$ y en los niños con dos o más situaciones de desprotección $(\mathrm{p}=$ $0,048)$. 
Tabla 4. Comparación de la presencia/ausencia de caries no tratada con el IMC y la situación de desprotección en el conjunto de niños de otros paises Aragón (España)

Otros países Latinoamericanos

\begin{tabular}{lcc} 
& $\frac{\text { Caries no tratada }(+)}{(n=24)}$ & $\frac{\text { Caries no tratada }(-)}{(n=151)}$ \\
Variables demográficas [n (\%)] & $12(50)$ & $66(43,7)$ \\
Varones & $12(50)$ & $85(56,3)$ \\
Mujeres & $15(62,5)^{*}$ & $37(24,5)$ \\
Edad 6-12 años & $9(37,5)$ & $114(75,5)$ \\
Edad 13-17 años & $5(20,8)^{*}$ & $10(6,6)$ \\
Generación 2 & $4(16,7)$ & $24(15,9)$ \\
Generación 1.75 & $12(50)$ & $76(50,3)$ \\
Generación 1.5 & $3(12,5)$ & $41(27,2)$ \\
Generación 1.25 & & \\
IMC [n (\%)] & $21(87,5)$ & $127(84,1)$ \\
Normal & $1(4,2)$ & $16(10,6)$ \\
Sobrepeso & $2(8,3)$ & $8(5,3)$ \\
Obesidad & $3(12,5)$ & $24(15,9)$ \\
Sobrepeso + Obesidad & & \\
Situación de desprotecclon [n (\%)] & & \\
Incapacidad parental de control de la & $3(12,5)$ & $65(43) *$ \\
$\quad$ conducta del menor & $7(29,2)$ & $26(17,2)$ \\
Maltrato físico & $6(25)$ & $18(11,9)$ \\
Negligencia fisica & & \\
Imposibilidad parental temporal o definitiva & $7(29,2)$ & $21(13,9)$ \\
para ejercer los deberes de protección & $3(12,5)$ & $16(10,6)$ \\
Maltrato psicológico & $3(12,5)$ & $14(9,3)$ \\
Abandono & $0(0)$ & $6(4)$ \\
Abuso sexual & $5(20,8)^{*}$ & $12(7,9)$ \\
Dos o más situaciones de desprotección & & \\
\hline
\end{tabular}

$* \mathrm{p}<0,05$

En la Tabla 5 se comparan las variables demográficas, el IMC y las situaciones de desprotección del grupo de niños ecuatorianos y el grupo de niños del conjunto de países latinoamericanos con caries no tratada. Excepto la existencia de una mayor prevalencia en los niños ecuatorianos de la generación $1.75(\mathrm{p}=0,016)$, no se observaron diferencias significativas.

Tabla 5. Comparación de la presencia de caries no tratada entre los niños ecuatorianos y el conjunto de otros países latinoamericanos en el sistema de protección de Aragón (España)

\begin{tabular}{|c|c|c|}
\hline & $\begin{array}{l}\text { Caries no tratada }(+) \\
\quad(n=15)\end{array}$ & $\begin{array}{l}\text { Caries no tratada }(+) \\
\quad(n=24)\end{array}$ \\
\hline \multicolumn{3}{|l|}{ Variables demográficas [n (\%)] } \\
\hline Varones & $3(20)$ & $12(50)$ \\
\hline Mujeres & $12(80)$ & $12(50)$ \\
\hline Edad 6-12 años & $9(60)$ & $15(62,5)$ \\
\hline Edad 13-17 años & $6(40)$ & $9(37,5)$ \\
\hline Generación 2 & $2(13,3)$ & $5(20,8)$ \\
\hline Generación 1.75 & $8(53,3)^{*}$ & $4(16,7)$ \\
\hline Generación 1.5 & $4(26,7)$ & $12(50)$ \\
\hline Generación 1.25 & $1(6,7)$ & $3(12,5)$ \\
\hline \multicolumn{3}{|l|}{ IMC [n (\%)] } \\
\hline Normal & $14(93,3)$ & $21(87,5)$ \\
\hline Sobrepeso & $0(0)$ & $1(4,2)$ \\
\hline Obesidad & $1(6,7)$ & $2(8,3)$ \\
\hline Sobrepeso + Obesidad & $1(6,7)$ & $3(12,5)$ \\
\hline \multicolumn{3}{|l|}{ Situación de desprotección [n (\%)] } \\
\hline \multicolumn{3}{|l|}{ Incapacidad parental de control de la } \\
\hline Maltrato físico & $6(40)$ & $7(29,2)$ \\
\hline Negligencia física & $2(13,3)$ & $6(25)$ \\
\hline \multicolumn{3}{|l|}{ Imposibilidad parental temporal o definitiva } \\
\hline para ejercer los deberes de protección & $5(33,3)$ & $7(29,2)$ \\
\hline Maltrato psicológico & $2(13,3)$ & $3(12,5)$ \\
\hline Abandono & $1(6,7)$ & $3(12,5)$ \\
\hline Abuso sexual & $0(0)$ & $0(0)$ \\
\hline Dos o más situaciones de desprotección & $3(20)$ & $5(20,8)$ \\
\hline
\end{tabular}

${ }^{*} \mathrm{D}<0.05$

\section{Discusión}

En los países de Latinoamérica la caries es un problema de salud pública debido a las altas prevalencias reportadas. ${ }^{13}$ Según la FDI el índice CPOD a los 12 años de edad es alto en Bolivia, Ecuador, Guatemala, Honduras, Panamá, Perú, Puerto Rico y República Dominicana. ${ }^{4}$ En Ecuador, en concreto, el estudio epidemiológico nacional de salud bucal en escolares menores de 15 años observó un índice CPOD de 0,22 a los 6-7 años de edad, de 2,95 a los 12 años y de 4,64 a $\operatorname{los} 15$ años. ${ }^{14}$

En la muestra estudiada de niños ecuatorianos la prevalencia de caries no tratada fue de 25,4\%. Esta proporción fue casi el doble que la observada en los niños del conjunto de otros países latinoamericanos, de los que el 32\% $(n=56)$ eran originarios de países con un índice CPOD alto según la FDI.

En España, la encuesta de salud oral realizada en 2015 mostró una prevalencia de caries no tratada en niños de 12 años de 14,6\%. ${ }^{15}$ En la Comunidad Autónoma de Aragón, los resultados de un estudio del programa de atención bucodental infanto-juvenil realizado en 2011 mostró una prevalencia de caries no tratada en niños de 12 años de 8,6\%. ${ }^{16}$ Estas proporciones también fueron significativamente inferiores a las observadas en los niños ecuatorianos.

En los niños ecuatorianos se observó una asociación positiva de la caries no tratada con el sexo femenino, mientras que en los niños del conjunto de otros países latinoamericanos se observó asociación positiva con el grupo de edad de 6-12 años y con los pertenecientes a la segunda generación, es decir, con los nacidos en España. Diversos estudios han puesto de relieve que la población en edad escolar que migró con su familia a países europeos o con el antecedente de migración de sus padres (segundas generaciones) presenta generalmente una prevalencia de caries superior a la de la población de acogida. La mayor prevalencia de caries en esta población parece estar relacionada con las dietas cariogénicas de los países de acogida, los cuidados dentales inadecuados, y las deficiencias nutricionales. ${ }^{2,17}$

Según la FDI la caries se debe principalmente a la exposición al azúcar. ${ }^{4}$ El sobrepeso y la obesidad en la edad pediátrica es cada vez más prevalente y los expertos lo vinculan, entre otros factores, al exceso de consumo de azúcar. ${ }^{5}$ Según esta asociación, los niños con sobrepeso y obesidad deberían tener una mayor prevalencia de caries. En este estudio no se observó diferencia en la prevalencia de sobrepeso y obesidad entre los niños ecuatorianos y el conjunto de niños de otros países latinoamericanos, y tampoco se observó asociación positiva entre la existencia de sobrepeso y obesidad y la presencia de caries no tratada en y entre ambas muestras. La ausencia de asociación entre 
la caries dental y el sobrepeso y obesidad sería acorde con lo observado en la mayoría de los estudios de revisión sistemática de la literatura y metaanálisis. ${ }^{18,20}$

Respecto a la relación entre la situación de desprotección que condujo a estos niños a su acogimiento en el sistema de protección de Aragón y la presencia de caries no tratada, en los niños ecuatorianos se observó una asociación positiva con la imposibilidad parental temporal o definitiva para ejercer los deberes de protección (por fallecimiento, encarcelamiento, enfermedad física, mental o toxicomanía incapacitantes o causas de naturaleza similar, junto a la ausencia de familiares que se hagan cargo del cuidado del menor), y en el conjunto de niños de otros países latinoamericanos se observó una asociación positiva con la existencia de dos o más situaciones de desprotección. Estas asociaciones serían acordes con los estudios que reconocen que la caries dental no tratada es un trastorno vinculado a la vulnerabilidad y deprivación sociofamiliar. ${ }^{21}$

\section{Conclusiones}

En la muestra de niños ecuatorianos acogidos en el sistema de protección de Aragón (España) por encontrase en situación de riesgo social:

- La prevalencia de caries no tratada fue alta y significativamente superior a la observada en los niños del conjunto de otros países latinoamericanos con la misma problemática y en los niños del país y la comunidad de acogida.

- No se observó asociación entre la presencia de caries no tratada y el estado nutricional de acuerdo con el IMC.

- La presencia de caries no tratada mostró asociación positiva con la situación de imposibilidad parental temporal o definitiva para ejercer los deberes de protección.

Consideramos que, además de los hábitos dietéticos cariogénicos nativos, la situación de desprotección y deprivación sociofamiliar es un factor esencial en la alta prevalencia de caries no tratada observada en estos niños.

\section{Referencias}

1. World Health Organization. Report on the health of refugees and migrants in the WHO European Region. No Public health without refugee and migrant health. Copenhagen: WHO Regional Office for Europe; 2018.

2. Oficina de Estadística de la Unión Europea (Eurostat). Population: demography, population projections, census, asylum \& migration - Overview. European Commission. Disponible en: https://ec.europa.eu/eurostat/web/population/overview
3. Instituto Nacional de Estadística (INE). Población extranjera por Nacionalidad, provincias, Sexo y Año (1998-2018). Ministerio de Economía y Empresa Gobierno de España. Disponible en: ht tps://www.ine.es/jaxi/ Tabla.htm? pa$\mathrm{th}=/ \mathrm{t} 20 / \mathrm{e} 245 / \mathrm{p} 08 / 10 / \&$ file $=03005 . \mathrm{px} \& \mathrm{~L}=0$

4. FDI World Dental Federation. El Desafío de las Enfermedades Bucodentales - Una llamada a la acción global. Atlas de salud bucodental. $2^{\mathrm{a}}$ ed. Ginebra: Federación Dental Internacional (FDI); 2015.

5. Moreno LA, Pigeot I, Ahrens W. Childhood Obesity: Etiology - Synthesis Part II. Etiological Factors. En: Moreno LA, Pigeot I, Ahrens W, editores. Epidemiology of Obesity in Children and Adolescents. Prevalence and Etiology. New York: Springer; 2011. p. 483-492.

6. Oliván G. Untreated dental caries is common among 6 to 12 -year-old physically abused/neglected children in Spain. Eur J Public Health. 2003;13(1):91-92.

7. Oliván Gonzalvo G. Elevada incidencia de caries no tratada en dientes permanentes entre adolescentes en riesgo social. An Esp Pediatr. 2002;57(3):270-271.

8. Boletín de Datos Estadísticos de Medidas de Protección a la Infancia. Observatorio de la Infancia. Ministerio de Sanidad, Consumo y Bienestar Social. Gobierno de España. Disponible en: http://www.observatoriodelainfancia.mscbs.gob.es/estadisticas/estadisticas/home.htm.

9. Instituto Aragonés de Servicios Sociales, Servicio de Atención a la Infancia y Adolescencia. Instrumento para la Valoración de la Gravedad de las Situaciones de Desprotección en Aragón. Zaragoza: Gobierno de Aragón- Departamento de Sanidad, Bienestar Social y Familia; 2014.

10. Checa J, Monserrat M. La integración social de los hijos de inmigrantes africanos, europeos del este y lati $\neg$ noamericanos: un estudio de caso en España. Uni $\neg$ versitas Psychologica. 2015;14(2):475-486.

11. Cole TJ, Bellizzi MC, Flegal KM, Dietz WH. Establishing a standard definition for child overweight and obesity worldwide: international survey. BMJ. 2000;320(7244):1240-1243.

12. World Health Organization. Oral Health Surveys. Basic Methods. Fifth Edition. Genève: WHO; 2013.

13. Martins Paiva S, Álvarez Vidigal E, Abanto J, Cabrera Matta A, López Robles RA, Masoli C, et al. Epidemio- 
logía de la caries dental en América Latina. Rev Odontopediatr Latinoam. 2014;4(2):13-18.

14. Raza X, Alvear A, Andrade R, Ayala E, Chilliquinga M, Luque I, et al. Estudio Epidemiológico Nacional de Salud Bucal en Escolares Menores de 15 años del Ecuador. Quito: MSP/OPS; 2010.

15. Bravo Pérez M, Almerich Silla JM, Ausina Márquez V, Avilés Gutiérrez P, Blanco González JM, Canorea Díaz E, et al. Encuesta de Salud Oral en España 2015. RCOE. 2016;21(Supl. 1): 8-48.

16. Santos Bistué C, Rubio Calvo E, García Felipe AI, Núñez Bielsa E. Estado de salud oral de los niños de 12 años en Barbastro antes y tras la instauración del PABIJ (Programa de Atención Bucodental Infanto-Juvenil de Aragón). Rev Eur Odontoestomatol. 2015;2:e1.

17. Valcárcel Soria R, Somacarrera Pérez ML. Estado de salud oral en los niños inmigrantes en España. Odontol Pediátr (Madrid). 2016;24(3):194-206.

18. Carson SJ. No consistent association found between dental caries and body mass index in children. Evid Based Dent. 2018;19(2):38-39.

19. Chen D, Zhi Q, Zhou Y, Tao Y, Wu L, Lin H. Association between dental caries and BMI in children: a systematic review and meta-analysis. Caries Res. 2018;52(3):230-245.

20. Ruiz Mena K, Trávez Pacheco S, Toalombo Puma O, Sotelo Garzon E, Armas Vega A. La obesidad en niños y adolescentes como factor desencadenante de caries dental, revisión bibliográfica. Rev Odontopediatr Latinoam. 2019;9(1):48-53.

21. Locker D. Deprivation and oral health: a review. Community Dent Oral Epidemiol. 2000;28(3):161-169.

Recibido: 17 de enero de 2020

Aceptado: 19 de abril de 2021 